\title{
SOBRE LA NATURALEZA DE LOS ESTUDIOS UNIVERSITARIOS
}

Francisco Muscará

Pontificia Universidad Católica Argentina (sede Mendoza) - UCA

\section{RESUMO}

O objetivo principal deste artigo é perguntarmos se os estágios históricos que surgiram na Universidade de cultura ocidental são verdadeiramente um processo de degradação e, em caso afirmativo, o que devemos fazer agora para recuperar, de alguma forma, sua ideia inicial: a busca desinteressada da verdade. Vamos rever as principais características do modelo fundado na Idade Média; na Universidade Humboltiana; na educação do cavalheiro Inglês; no modelo napoleônico e no modelo norte-americano. Finalmente, faremos uma breve referência às universidades argentinas que sempre priorizaram a formação de profissionais e, por último, buscaremos nas recentes propostas da UNESCO algumas ideias que nos permitem pensar um sistema de ensino superior, a partir de uma atitude científica, procurando melhorar a qualidade das instituições que oferecem formação profissional.

Palavras-chave: Educação, História da Educação, Universidade.

\section{ABSTRACT}

The main objective of this paper is to ask whether the historical stages that emerged in the University of Western culture are truly a process of degradation and, if so, what should we do to recover, somehow, the original idea: the disinterested pursuit of truth. Let's review the main features of the model established in the Middle Ages; at the Humboltiana University; the education of English gentleman; in the Napoleonic model and the American model. Finally, we will make a brief reference to the Argentine universities, that have always prioritized the training of professionals and, finally, the recent proposals of UNESCO, some ideas that allow us to think of a higher education system, from a scientific attitude, seeking to improve the quality of the institutions that offer vocational training.

Keywords: Education, History of Education, University

\section{RESUMEN}

El objetivo principal de este documento es preguntarnos si las etapas históricas por las que atravesó la Universidad en la cultura de Occidente constituyen verdaderamente un proceso de degradación y, en ese caso, qué tendríamos que hacer ahora para rescatar, de alguna manera, su ideal inicial: la búsqueda desinteresada de la verdad. Pasaremos revista a las principales características del modelo fundacional en la Edad Media; de la Universidad Humboltiana; de la Educación del gentleman inglés; del modelo napoleónico y del modelo norteamericano. Terminaremos con una breve referencia a las universidades argentinas que siempre priorizaron la formación de profesionales y, por último, buscaremos en las recientes propuestas de la UNESCO algunas ideas que nos permitan pensar en un sistema de educación superior que, desde una actitud académica, busque mejorar la calidad de las instituciones que ofrecen carreras de formación profesional.

Palabras-clave: Educación, Historia de la Educación, Universidad. 


\section{Introducción: ¿Degradación de la Universidad?}

Bajo este título, algunos profesores relatan la historia de la universidad en la vida de Occidente ${ }^{1}$. Es que para ellos la esencia de la vida universitaria, su finalidad, no debe ser otra que "la investigación y transmisión o docencia de la verdad en un nivel superior de la ciencia o de-velación de sus causas en todas sus manifestaciones particulares y en su visión sapiencial de la Filosofía y de la Teología cristianas en la actual providencia del hombre que las integra o unifica en el plano fundamental de sus últimas causas" (DERISI,1980, Naturaleza y vida de la universidad, p. 20).

Según ellos, la Universidad no es una institución directamente ordenada a la acción sino que su naturaleza es eminentemente teorética o especulativa. El carácter académico, propio de los estudios universitarios consiste en que los distintos sectores de la ciencia y de la técnica se realicen con un deseo desinteresado de descubrir y encontrar la verdad. También en el obrar moral, es decir en todos los aspectos de la actividad práctica humana, la Universidad considera cuáles deben ser los principios que guíen las acciones humanas. Este es el espíritu que debe animar a toda la tarea universitaria porque la Universidad estudia la economía pero no es una empresa; estudia las técnicas en sus múltiples direcciones pero no es una fábrica; estudia la política pero no es un partido; estudia teología pero no es una religión.

Sólo en esta actitud teorética, en esta independencia respecto de cualquier fin utilitario, puede fundarse la libertad académica. Por eso, dice Caturelli (1964): "La Universidad es ciencia que busca la sabiduría y el movimiento hacia la unidad (versus unum) es idéntico con ella; luego, toda la vida académica es, en el fondo, un 'versus sapientiam', hacia la Sabiduría, porque ésta se sigue de la contemplación de toda Verdad... La Universidad, en cuanto comunidad académica, se orienta hacia la contemplación” (CATURELLI, 1964, LA UNIVERSIDAD, p. 54).

Sin embargo, a ese modelo original de la Universidad medieval que buscaba realizar la síntesis entre las verdades de fe y las de razón puesto que reconocía a Dios como el primer principio de todas las cosas y primera causa del orden natural. A esa universidad medieval le seguirá la Universidad del Renacimiento que, dejando de lado la Revelación, pondrá su confianza en la razón humana como último fundamento de explicación de todo lo real. El cisma del cristianismo y, por consiguiente, de la unidad cultural; el inicio de la expansión de los estados nacionales y el progreso del pensamiento experimental determinó, en los albores de la Edad Moderna, la crisis de la Universidad Medieval.

La Universidad filosófica, antropocéntrica, conservó la unidad que establece la Filosofía con relación a las ciencias particulares pero perdió la posibilidad de alcanzar la universalidad del saber y de plantearse el problema del último fundamento del universo ya que "sólo puede haber teoría en pleno sentido; sólo es realizable como actitud, cuando se considera al mundo como creación... es el culto lo que hace posible y constituye desde dentro esa libertad fáctica y jurídica de la 'scholé', que es a la vez ocio y escuela" (PIEPER, 1979)

A partir del desarrollo de las ciencias particulares surgirá un nuevo tipo de universidad, sin criterio ordenador de los estudios. Diversas personas estudiarán por su cuenta distintos asuntos: Bacon, Hobbes, Locke, Descartes, Pascal, Spinoza... Afirma Villey: "En lugar de estudiar la naturaleza, de ensayar y comprender su estructura y sus caracteres esenciales, precisamente porque ellos construyen su doctrina demasiado solitariamente, los autores modernos se complacen en sus ideas; y ellos construyen sobre estas ideas sistemas, como los geómetras embobinan sobre axiomas séquitos de teoremas: sistemas variados, que forman tantas doctrinas particulares como obras de arte 
personales. Ahora el mundo sería construido o bien a partir de conceptos llamados innatos, como el 'cogito' de Descartes, o bien de ideas que se dicen sacadas de la experiencia física, moral, religiosa, pero de una experiencia muy estrecha, propia de la especialización científica de cada autor. Hay tantos sistemas como profesores." (MONTEJANO, 1979, p. 98).

Al prescindir de los estudios teológicos y filosóficos, los universitarios se vuelven intelectuales especializados en un estrecho sector de la realidad; aún persiguen la búsqueda de la verdad pero han perdido la posibilidad de alcanzar la universalidad del saber y obtener la unidad de conocimientos dispersos. ${ }^{2}$

A la Universidad Cientificista siguió la Universidad Profesional y aquí se nota claramente el cambio de finalidad. Ya no interesa tanto aprender cuanto alcanzar un título que habilite para trabajar. La nueva actitud fue descripta por Piero Calamandrei (1960): "La instrucción no se busca ni se desea por el bien que da al espíritu; la buscan de prisa, sin amor y sin fe, los que ven en ella el único medio para obtener del Estado aquel milagroso carnet denominado, según los casos, títulos de bachiller, de licenciado o de doctor, que da derecho al que lo posee de comer pan por toda la vida... y no siendo lo que más importa aprender sino apoderarse del documento en el que está oficialmente escrito que se ha aprendido, se comprende fácilmente que en la aplicación de la ley del mínimo esfuerzo, los llamados estudios se hayan reducido a una serie de estratagemas encaminados a obtener sin fatiga el título" (MONTEJANO, 1979, p. 100).

El objetivo principal de este documento es preguntarnos si estas etapas históricas por las que atravesó la Universidad en la cultura de Occidente constituyen verdaderamente un proceso de degradación y, en ese caso, qué tendríamos que hacer ahora para rescatar, de alguna manera, su ideal inicial: la búsqueda desinteresada de la verdad. Pasaremos revista a las principales características del modelo fundacional en la Edad Media; de la Universidad Humboltiana; de la Educación del gentleman inglés; del modelo napoleónico y del modelo norteamericano. Terminaremos con una breve referencia a las universidades argentinas que siempre priorizaron la formación de profesionales $\mathrm{y}$, por último, buscaremos en las recientes propuestas de la UNESCO algunas ideas que nos permitan pensar en un sistema de educación superior que, desde una actitud académica, busque mejorar la calidad de las instituciones que ofrecen carreras de formación profesional.

\section{Origen de la vida universitaria em la Edad Media ${ }^{3}$}

El antecedente más próximo de las universidades europeas fueron las escuelas episcopales o catedralicias que se caracterizaban por revalorizar la filosofía antigua. En estas escuelas se enseñaban las artes liberales ${ }^{4}$ : el trivium: gramática, retórica y dialéctica; y el quadrivium: aritmética, geometría, música y astronomía de acuerdo con la organización que les había dado Boecio ${ }^{5}$. No obstante, estas escuelas no configuraban una Universidad porque: a) las facultades no se integraban entre sí para constituir un cuerpo; b) los cursos no se desarrollaban con regularidad y de acuerdo a programas; c) los miembros de la comunidad no pertenecían a ella de una manera estable.

Las universidades nacieron en Europa en los albores del siglo XIII debido a que se conjugaron una serie de circunstancias: la organización gremial de la sociedad y el influjo de la ciencia y la teología árabes cuyo conocimiento fue uno de los resultados de las Cruzadas que produjeron un intercambio cultural entre Oriente y Occidente. La organización gremial, en la Edad Media, permitía la constitución de sociedades de una misma clase social o profesión, por eso, así como existían corporaciones de artes y oficios, 
la universitas era una comunidad de maestros y discípulos cuya ocupación era el estudio, la enseñanza y la búsqueda de la sabiduría. Tenían por finalidad el deseo de desarrollar el saber y extenderlo por todo el mundo; organizar las carreras indispensables a la sociedad pero, sobre todo, ofrecer un servicio a Dios puesto que la mayoría de ellas estaban amparadas por la Iglesia.

Al comienzo se denominaron 'studium generale' para dar a entender que estaban abiertas a todos los jóvenes de cualquier nacionalidad y que allí se cultivaban todas las ciencias. Como los títulos alcanzados por los graduados eran reconocidos en todas partes, las universidades tenían que ser creadas o reconocidas por el Papa (que tenía poder sobre todos los países cristianos) o por el Emperador. Como ejemplo, de acuerdo a su origen, podemos citar:

- Sin carta de fundación (por derecho consuetudinario): Oxford

- Con carta de fundación pontificia: Roma (1303) y Colonia (en la que estudió Santo Tomás de Aquino)

- Con carta de fundación imperial: Salamanca (fundada por Alfonso IX)

- Con doble carta fundacional: la Universidad de Viena

No obstante, los títulos y privilegios suponían siempre el reconocimiento de asociaciones culturales pre-existentes que permitían la fundación y el futuro desarrollo de la nueva institución. Los principales privilegios concedidos a los universitarios eran la 'licentia docendi' (autorización para enseñar en todas partes, lo cual suponía: "ser home de buena fama y de buenas maneras", "tener buen entendimiento del testo e de la glosa y desembargada lengua para mostrarla" (Partida IX de Alfonso El Sabio); el derecho de reunirse en asamblea y elegir autoridades; la exención del servicio de armas; la dispensa de algunos impuestos y contribuciones y, a instancias del Rey Felipe Augusto (1200), el fuero académico, gracias al cual algunos delitos cometidos por maestros o estudiantes sólo podían ser juzgados por los mismos universitarios.

Según Durkheim: "Lo primero que debe admitirse sin reservas en el período de la universidad medieval, es su admirable fecundidad en materia de organización escolar... En un punto determinado del continente europeo, de un amplio cuerpo enseñante, anónimo, impersonal, perpetuo por consiguiente, que comprendía centenares de maestros y millares de estudiantes, asociados todos a la misma obra y sometidos a la misma regla; organización de ese cuerpo de forma que fuese, en la medida de lo posible, representativo de todas las disciplinas humanas; creación, dentro de este mismo sistema, de órganos secundarios que, con el nombre de facultades, corresponden a las diferentes especialidades del saber... institución de grados que jalonan la vida escolar y marcan sus etapas; institución de exámenes que protegen el acceso a estos grados; institución, para terminar, de planes de estudios que fijan al estudiante los conocimientos que debe adquirir durante cada una de estas fases, y a los maestros las materias que deben enseñar: tales son las principales novedades que aparecen en el espacio de dos o tres siglos" (Cit. por Krotsch en Educación superior y reformas comparadas, p. 63. El destacado es nuestro)

Universidad de París: A comienzos del siglo XII, París se convierte en la capital del reino de los Capetos y en lugar de residencia de la Corte. La escuela de París ejerce una fuerte atracción para los jóvenes del reino y de los territorios vecinos. En 1208 fue reconocida por el Papa Inocencio III como universitas magistrorum et scholarium Parisius commorantium $^{6}$; es probable que ello ocurriera porque la mayoría de los alumnos y de los maestros eran clérigos,

Poco a poco, los maestros que enseñaban en Chartres, en San Victor y en NotreDame se encontraron en una situación análoga a la de los maestros de una industria o de un comercio y sintieron la necesidad de organizarse corporativamente ${ }^{7}$. Como ocurría en las 
demás profesiones con la obra maestra que debía producir el oficial, el estudiante que terminaba su formación tenía que desarrollar una lección inaugural en presencia de un maestro que diese fe de su capacidad. Esta ceremonia, en la que se entregaba a los graduados las insignias de su nueva profesión, significaba que sólo los maestros en ejercicio tenían poder para conceder el derecho a enseñar. Finalmente, después de que muchos maestros y estudiantes emigraron a Toulouse por causa de graves disturbios, la Universidad de París recibió su Carta Magna en la Bula "Parens scientiarum"; en ella se autorizaba a la corporación a legislar sobre cursos, estudios y grados y se confirmaba su sometimiento a la autoridad eclesiástica.

La Universidad se organizó en cuatro facultades: la de Artes, Derecho, Medicina y Teología. Aunque la Universidad de París era famosa por sus estudios de Teología porque allí se explicaban las "Sentencias" de Pedro Lombardo y allí enseñaron maestros como San Buenaventura y Santo Tomás de Aquino que buscaban armonizar las verdades de razón con las verdades de $\mathrm{fe}^{8}$. El conjunto de los estudios estaba dominado por un sistema de grados y exámenes. Este régimen de exámenes respondía al hecho de que el gremio de estudiantes y maestros, al igual que otras corporaciones, era un cuerpo cerrado que sólo abría sus puertas a aquellos que demostraban poseer las aptitudes exigidas. Los grados eran las sucesivas etapas que había que pasar hasta vencer la resistencia de la corporación. Hasta los quince años el joven sólo debía estudiar; después de esa edad, a la vez que seguía la carrera hacia el doctorado, podía empezar a enseñar. El paso de una etapa a otra estaba determinado por el bachillerato. El grado por excelencia, que constituía el coronamiento del aprendizaje, era el de Magíster en la Facultad de Artes; equivalente al grado de Doctor en las otras facultades; quien alcanzaba este título gozaba de plenitud de derechos en la comunidad universitaria.

El año escolar duraba unos nueve meses y los métodos de la Pedagogía Universitaria se articulaban en tres tipos de actividades: La lectio que consistía en la lectura de libros que eran considerados básicos. Luego, para avanzar en la comprensión de las partes más difíciles, tenían lugar las repeticiones (explicaciones y comentarios) que se realizaban en forma de diálogo entre los maestros y los estudiantes. Por último, la disputatio en la que un maestro pronunciaba una conferencia y se sometía a las preguntas de los demás profesores que adherían o se oponían a la tesis propuesta. La disputatio era un expediente magnífico para desarrollar la destreza dialéctica, pero no pocas veces fomentó el formalismo de sutilezas insustanciales.

La facultad más numerosa era la de Artes porque constituía una especie de curso de selección en la que se preparaba a los estudiantes para el ingreso a otras facultades. La facultad de Artes gozaba de algunos privilegios: a ella debía pertenecer el Rector de la Universidad y ella podía expulsar a algún alumno sin necesidad de consultar con otras facultades.

Siguiendo la tradición de las escuelas monacales, la enseñanza universitaria fue gratuita en el comienzo, pero cuando los maestros no fueron únicamente los clérigos y necesitaron vivir de su trabajo profesional, los estudiantes debieron abonar aranceles diferenciados según el tipo de estudios que seguían. Institucionalmente, las universidades tardaron mucho tiempo en tener recursos propios.

Universidad de Bolonia: Nació como tributaria de tres sistemas: el de la escuela catedralicia donde se enseñaban las Artes; las escuelas municipales, dedicadas al estudio del Derecho Civil y las escuelas monásticas, para la enseñanza del Derecho Canónico. El aprendizaje de la jurisprudencia se documentaba: para el derecho profano en la colección de Derecho Romano redactada por Justiniano y para el Derecho Eclesiástico en la compilación de Actas de los Concilios y las decretales pontificias. 
A diferencia de París que nació como iniciativa de los maestros, Bolonia se organizó en torno a la corporación de los estudiantes, quienes no sólo pagaban a los maestros por sus clases sino que además definían las características de su propia instrucción?. El primer documento que reconoció oficialmente la existencia de un grupo de estudiantes y maestros en Bolonia data de 1158. En él Federico Barbarroja concedió jurisdicción especial, escolar y eclesiástica a los extranjeros residentes en la Ciudad.

Los maestros eran ciudadanos boloñeses y estaban sujetos a las ordenanzas municipales; por su parte, el número de estudiantes crecía debido a los privilegios que se les concedían. La turbulencia juvenil, su falta de respeto hacia las autoridades, su apasionada intervención en las luchas políticas y sus frecuentes desmanes motivaron una propuesta de abolición de los privilegios pero esa iniciativa no prosperó. En el último tercio del siglo XIII (1275), las relaciones entre la Universidad (dependiente del Papa) y el Municipio fueron pacíficas; ello favoreció el normal desarrollo de la vida académica. En 1288 se organizó la Facultad de Artes y la de Medicina. En esta última, la Universidad se adelantó a Salerno practicando la disección de cadáveres para estudiar la anatomía del cuerpo humano.

Para describir los momentos de apogeo y de decadencia de la Universidad de Bolonia dice Mondolfo (1957): "Cuando la elección de los maestros ya no la hacen los estudiantes, interesados en buscar a los mejores sino la Comuna, prevalecen, especialmente en el siglo XIII, estrechos criterios municipales e intereses locales; los maestros elegidos de este modo, tienden a cristalizarse en la rutina y a dejarse dominar por los intereses profesionales. El nivel de la enseñanza decae; disminuye la atracción, especialmente para los estudiantes foráneos; y cooperan en esta tendencia negativa los conflictos entre los privilegios estudiantiles y el orden de la ciudad que provocan a veces secesiones de estudiantes, como la de 1292 que da lugar a la creación de la Universidad de Padua; y también el florecimiento de otros estudios produce a veces la emigración de maestros y escolares" (Cit. por CIRIGLIANO, op. cit., p. 37).

Salerno y Montpellier: En la Edad Media se consideraba a la Medicina como la física de lo concerniente al hombre en tanto que tiene materia y movimiento: anatomía y fisiología. En Salerno (Italia), debido a la existencia de los Monasterios de La Cava y de Montecassino, se tradujeron abundantes manuscritos clásicos; fue el modo como la Europa medieval conoció los estudios de medicina de la antigüedad. La enseñanza acudía a las obras de Hipócrates (-V A.C.) y de Galeno (siglo II) y a la enciclopedia médica del filósofo árabe Avicena (siglo XI).

En Salerno se fundaron hospitales para extranjeros que atendían a las personas que transitaban por la Ciudad. Los médicos transmitían sus conocimientos de un modo individual y asistemático. Fue un monje de Montecassino quien tradujo tratados de medicina árabe y elaboró una serie de manuales que se usaron en las escuelas hasta el siglo XV.

Hacia el siglo XIII, la Escuela de Salerno había cumplido una gran misión: compilar, elaborar y adaptar a las necesidades académicas la medicina antigua clásica y la paleo-árabe. El "antidotario" (colección de recetas médicas, formularios y diagnósticos) recibió su forma definitiva. A finales del siglo surgió un grupo (los cirujanos) que quiso difundir una orientación más práctica de los estudios.

Sin embargo, Salerno comenzó a declinar a favor de Montpellier. Esta Universidad se organizó entre 1180 y 1220 y reglamentó todo lo necesario para conceder títulos de licenciatura. La Medicina se separó del conjunto de las Artes liberales y alcanzó autonomía científica. Hacia 1240 fueron confirmados los estatutos de esta Universidad que ya había aprobado el legado pontificio, Cardenal Conrado de Urach. 
Oxford: Esta Universidad tuvo su origen en la escuela catedralicia de la ciudad cuando regresaron estudiantes ingleses que estudiaban en París porque fueron expulsados de la universidad (1167). Se destacó por el estudio de las ciencias naturales; la institución se organizó como una federación de "colleges".

Pauline Walton (1965), describe algunas tradiciones que se cultivaban en la vida de esta casa de estudios: “...el sistema de enseñanza por medio de tutores estudiantiles sigue siendo el básico. Los estudiantes, vistiendo sus togas, deben cenar en el hall varias veces por semana... Allí, los "dons" (profesores residentes) se sientan a la Gran Mesa y la cena transcurre con cierta ceremonia. Los estudiantes no pueden estar fuera después de la medianoche, con excepción de los 'Christ Church', que disponen de veinte minutos después del toque de queda... De noche, por las calles de la ciudad -en las que resuenan las campanas de sus muchas iglesias y relojes- se pasea el censor, que viste toga con vueltas de terciopelo. Lo acompañan dos policías universitarios apodados 'bull-dogs'. Si encuentran a un estudiante comportándose mal, le preguntan el nombre y a qué colegio pertenece y a la mañana siguiente el joven tiene que enfrentarse con el decano de la institución”.

Por su parte, en un reportaje que se realizó al estudiante Ian Jones sobre el régimen de estudios de Oxford, respondió: "El período lectivo consta de tres períodos de ocho semanas y de vacaciones se tienen seis semanas para Navidad y para Pascua y tres meses en el verano. A Uds. les sorprenderá que tengamos más vacaciones que período lectivo, pero gran parte de nuestro estudio debe hacerse durante las vacaciones. Debemos hacer lectura y trabajo general básico... tal es así, que a la entrada de cada período tenemos pruebas para verificar si uno ha trabajado o no durante las vacaciones... En cuanto a clases, Oxford y Cambridge están basados en un sistema muy peculiar: la Universidad en sí está dividida en colleges. Cada alumno tiene un tutor, hay alrededor de uno cada quince o veinte alumnos. El alumno debe hablar con este tutor una o dos veces por semana y éste le dice qué es lo que debe estudiar y le da trabajo para hacer. Hay lecturas (conferencias) de la Universidad, pero uno no está obligado a asistir. Cada colegio tiene sus tutores que son doctores con diversas distinciones... Se tiene un examen final al terminar el primer año y después no hay más exámenes hasta el tercer año... Los exámenes finales son muy bravos, son escritos y se toman ocho o nueve exámenes de tres horas en cuatro días..."10

La Sorbona: Fuera del ámbito académico, para ordenar la vida extraescolar, los universitarios se organizaron en Naciones que eran agrupaciones encargadas de proveer todo lo relativo al alojamiento, la comida y las diversiones de los estudiantes. En los hospitia convivían maestros y alumnos que provenían del mismo lugar. Estos alojamientos no tenían carácter oficial y eran sostenidos por jóvenes que podían pagar esos servicios. Ellos mismos elegían al responsable de la comunidad.

En el siglo XIII nacieron los Colegios Mayores que eran residencias organizadas para estudiantes pobres. Estos "colegios" proporcionaban a los becarios una vida disciplinar adecuada y la posibilidad de asistir a clases de repaso. Uno de los más importantes, que dio origen a una Universidad, fue el que fundó Roberto Sorbón (1257).

En los Colegios Mayores se difundió la práctica de las cuestiones disputadas; los becarios discutían todas las semanas bajo la dirección del Rector del Colegio. Luis Vives (1531) decía que se disputaba en todo tiempo y lugar, durante las comidas y después de ellas, en público y en privado. No obstante, el diálogo que requería exposición de la tesis y argumentos a su favor, planteamientos de las objeciones y solución de las dificultades, no consistía en una charla baladí. El profesor guiaba los debates e iluminaba los caminos para poner orden en las discusiones. 
A partir de París, Bolonia y Oxford, durante el siglo XIII se multiplicaron las instituciones por toda Europa: Palencia (1212); Salamanca (1220); Padua (1222); Nápoles (1224); Toulouse (1229). En todas se dieron problemas entre estudiantes y profesores; problemas de los universitarios con los habitantes de los lugares; conflictos de poder entre la Iglesia y el Imperio, etc. pero, a pesar de todas esas dificultades, la universidad medieval trató de mantenerse firme en sus ideales y nos legó como herencia: la preocupación por buscar la verdad; la autonomía frente a los poderes políticos y el diálogo entre los intelectuales. Por eso, Alfonso X (el Rey Sabio) de España prescribió sobre el trato que debían recibir los universitarios: "De buen ayre, e de fermosas salidas, deue ser la Villa, do quisieren establecer el Estudio, porque los Maestros que muestran los saberes, e los Escolares que los aprenden, biuan sanos en el, e puedan folgar, e recibir plazer en la tarde, quando se levantaren cansados del estudio. Otrosi deue ser abondada de pan, e de vino, e de buenas posadas, en que puedan morar, e pasar su tiempo sin gran costa. Otrosi dezimos, que los Ciudadanos de aquel logar, do fuere fecho el Estudio, deuen mucho guardar e honrrar a los Maestros, e a los Escolares, e a todas sus cosas" (Ley II de las Partidas... Cit. por MONTEJANO, op. cit., p. 89)

\section{Salamanca y las Universidades Hispanoamericanas}

No podremos comprender la vida universitaria hispanoamericana sino conociendo la estructura y organización de la Universidad de Salamanca (la Atenas de España) puesto que todas ellas reprodujeron en América, de manera más directa o indirecta, las líneas estructurales básicas del modelo salmantino.

Su fundación se atribuye a Alfonso IX, Rey de León (1188-1229). Sin embargo, es a Alfonso X, El Sabio (1226-1284), a quien la Universidad de Salamanca le debe su mayoría de edad. Este Rey ocupa un puesto destacado en la historia de la pedagogía occidental porque promovió la ordenación jurídica de aspectos importantes de la educación e impulsó la fundación de instituciones culturales en Toledo y en Murcia. A él se debe el Código de las Siete Partidas y la fundación de la Universidad de Sevilla (1254).

El Papa Alejandro IV otorgó confirmación oficial a los estudios por medio de la Bula "Inter Ea" (1255). Desde entonces, la Universidad recibió múltiples favores de los Pontífices y sus maestros, además de gozar 'licentia docendi' en otras universidades, fueron reconocidos con títulos de nobleza. Privilegios, inmunidades y exenciones eran el flanco institucionalizado de la defensa de una libertad vital para esta institución: la de pensar $^{11}$.

Como se lee en los estatutos, que era la norma suprema, el propósito de la Universidad no era sólo instruir, enriquecer la inteligencia, sino también educar, formar la voluntad, porque en la Universidad no sólo es razón se aprendan letras sino también virtud y buenas costumbres y composición. De allí que ordenen las Partidas: "los escolares finquen asosegados en sus posadas, et puñen de estudiar, et de aprender et de facer vida honesta y buena, ca los estudios para eso fueron establecidos" (Cit. por RODRÍGUEZ CRUZ en La Universidad en la América hispana, p. 22)

La máxima autoridad, cabeza de la Universidad, era el Rector aunque su poder estaba limitado porque en materia docente debía consultar al claustro de los consiliarios y en materia económica era asesorado por los definidores. En estos consejos participaban estudiantes y profesores por eso podemos decir que en la Universidad de Salamanca el llamado "cogobierno" ya se practicó desde la primera mitad del siglo XV. De esa manera, los universitarios vivían en un clima democrático y se formaban en el sentido de responsabilidad porque participaban en el gobierno de la institución; incluso en una 
época tuvieron derecho a voto en las clases de oposición para postular a las cátedras. No obstante, el claustro pleno era el supremo consejo resolutivo, el que trataba sobre los problemas más graves e importantes de la universidad, y legislativo en caso de reforma de los estatutos.

El método de enseñanza de Salamanca era variado, armonizaba el dictado de las materias con las disputas y demás ejercicios escolares aunque las clases consistían, casi siempre, en la explicación de un texto. Se atribuye al maestro Francisco de Vitoria la innovación de dictar apuntes a los alumnos. La facultad de medicina tenía una enseñanza de carácter eminentemente práctico y los catedráticos debían procurar cadáveres para hacer las disecciones.

El maestrescuela era el juez de la corporación, encargado de velar por el estudio, el orden y las buenas costumbres de los estudiantes. El debía examinar y decidir todas las causas civiles y criminales de los universitarios. Se les exigía, incluso, cierta austeridad en la vestimenta por eso se prohibían los colores vivos y el uso de joyas. Sin embargo, junto con el rigor de la disciplina y las exigencias del estudio, los estudiantes de Salamanca supieron generar ratos de juegos, novatadas, estudiantinas y tunas.

La costumbre de fundar universidades en conventos y en colegios tuvo auge en Hispanoamérica porque estas fundaciones resultaban más económicas a la Corona y ayudaban a satisfacer las necesidades del momento: sociales, administrativas, judiciales y eclesiásticas. Las universidades de San Marcos de Lima (1551) y de México (1551), precedidas por la de Santo Domingo (1538), pronto se constituyeron en las mayores del continente que reproducían en estas tierras el espíritu de Salamanca. La estructura y organización salmantina, toda su normativa y sus prácticas pedagógicas fueron proyectadas a las universidades hispanoamericanas. Es cierto que no podía realizarse una reproducción total porque el profesorado era escaso y las rentas exiguas pero poco a poco estas instituciones ejercieron su liderazgo y orientación sobre los nuevos centros de estudio que se fundaban ${ }^{12}$.

Felipe II, El prudente, estableció por Ley la jurisdicción universitaria y mandó que los rectores de Lima y de México "tengan jurisdicción en los doctores, maestros y oficiales de ellas, y en los lectores, estudiantes y oyentes que a ellas concurriesen, en todos los delitos, causas y negocios criminales que se cometieren e hicieren dentro de las escuelas de las universidades, en cualesquiera manera tocantes a los estudios... y en los delitos que se cometieren fuera de las escuelas, si fuere negocio tocante o concerniente a los estudios, o dependiente de ellos... porque el principal fin porque les concedemos esta jurisdicción es la reformación de vida y costumbres de los estudiantes, y que vivan corregidos y virtuosamente, para que mejor puedan conseguir la pretensión de sus letras" (Cfr. Recopilación de leyes de los Reinos de las Indias. Cit. por MONTEJANO, op. cit., p. 95).

En conclusión, podemos decir que aunque la función principal de las primeras universidades era la docencia puesto que las instituciones estaban más preocupadas por retener y transmitir conocimientos ya adquiridos, todas ellas contribuyeron a elevar el nivel cultural de la población y a formar maestros más capacitados. Por eso afirmó Giner de los Ríos (1876): "La Universidad fue una de las grandes fuerzas de la Edad Media, la única que representaba a la sazón la cultura superior del espíritu, cuando no había otros cuerpos científicos, ni imprenta, ni periódicos, ni revistas... Así intervenían en el gobierno de los Papas, de los Reyes, en todo... Por otra parte, su organización republicana, el ejercicio del sufragio, las frecuencias de las asambleas deliberativas, hacían de la universidad una escuela de libertad y un obstáculo contra la obediencia pasiva...” (Cit. por LARROYO, op. cit., p. 292). 


\section{La Universidad Alemana y el modelo Humboldtiano}

La derrota de Jena (1806) y la invasión de Napoleón a Alemania determinó que Fichte (1762-1814) se preocupara de organizar un sistema educativo que promoviera el sentimiento nacional de los habitantes. En sus Discursos a la Nación Alemana estableció los lineamientos de su propuesta. Allí afirmó que el Estado es el supremo sostén de la cultura y de la libertad y que, por eso, toda educación debe ser pública: "Reunir a los alemanes en un todo cuyos miembros estén animados de un mismo espíritu es el fin de la educación nacional". Los alemanes deben ser los fieles guardianes de los preciados tesoros de la humanidad y los sostenes del progreso cultural. En ese contexto, poco después, se preocupó de organizar la Universidad.

Inspirada por Fichte (que fue su primer Rector) Humboldt y Schleiermacher, la Universidad de Berlín (1810) desarrolló un modelo propio centrado en el sentimiento nacional y el desarrollo del espíritu. Esta Universidad fue concebida al servicio de la ciencia y de la investigación pura. Por su parte, Schleirmacher dirá que los problemas de la educación están condicionados por las exigencias morales, los avances de la ciencia y las formas de la experiencia estética, es decir, por la cultura de cada sociedad.

De esa manera se dejó de lado el ideal de la Universidad Medieval que Santo Tomás describió en la Suma Contra Gentiles: "La norma de orden y gobierno de cuanto se ordena a un fin se debe tomar del mismo fin; porque en tanto una cosa está perfectamente dispuesta en cuanto se ordena convenientemente a su propio fin, pues el fin es el bien de cada cosa. Así, vemos que en las artes, una, a la que atañe el fin, es como la reina y gobernadora de las demás: la medicina, por ej., impera y ordena a la farmacia, porque la salud, que es el objeto de la medicina, es el fin de todos los medicamentos confeccionados en farmacia. Y lo mismo sucede con el arte de navegar respecto de la industria naval, y con el militar respecto de la caballería, y de todas las otras ramas. Las artes que imperan a otras se llaman arquitectónicas o principales. Por esto sus artífices, llamados arquitectos, reclaman para sí el nombre de sabios. Mas como dichos artífices se ocupan de los fines de ciertas cosas particulares y no miran al fin universal de todas las cosas, se llaman sabios en esta o en otra materia. En cambio, se reserva el nombre de sabio con todo su sentido únicamente para aquellos que se ocupan del fin del universo, principio también de todos los seres. Y así, según el Filósofo, es propio del sabio considerar las cosas más altas" (I,1. El destacado es nuestro). Por encima del desarrollo de los saberes particulares, el objetivo académico principal es contemplar la verdad del primer principio y juzgar de las otras verdades.

Por su parte, Humboldt dirá que el principio esencial de la vida universitaria debe ser la ciencia tal y como existe, en toda su pureza. Por eso, junto con las escuelas y facultades que se dedican a transmitir el saber ya constituido deben existir Institutos que se consagren a la investigación y al desarrollo de las ciencias. Desde entonces la docencia y la investigación han sido consideradas funciones propias de la vida universitaria pero la idea unitaria de la Universidad comenzó a diluirse en el trabajo aislado de un conjunto de facultades, escuelas y organismos.

Puesto que la enseñanza universitaria exige la reelaboración y el análisis crítico de las conclusiones científicas, los estudiantes tendrán que ser educados no para reproducir sino para formarse en un juicio independiente; no se tendrán que limitar a recitar lecciones sino que deberán juzgar y redactar trabajos. Las didácticas de nivel superior tendrán que promover la relación directa de los estudiantes con la vida de la cultura e incentivar en ellos el estudio autónomo que supone la actitud de indagación. 
Según Nassif (1984), el modelo de Universidad plenamente académico quiso ser revivido por Kart Jaspers (1946), después de la caída de Alemania en la Segunda Guerra, pero eso fue imposible debido a la aceleración de la revolución científica y técnica y a las importantes transformaciones sociales y políticas que se produjeron.

\section{La “idea de Universidad" en John Newman}

Los valores espirituales que dieron a la Universidad Inglesa un carácter inconfundible fueron analizados en "The idea of a University" (1852) por el Cardenal Newman. Es más, en esas ideas se inspiraron un grupo de intelectuales de Dublín para fundar la Universidad Católica de Irlanda, única alternativa válida a otros centros de estudios superiores no confesionales que existían en aquella región. Parece que la Iglesia de Roma quería reproducir en Irlanda el modelo de la Universidad de Lovaina que había sido reactivada hacia 1830 como un centro universitario católico de buena calidad.

A juicio de Newman, lo específico de la institución universitaria es la universalidad. Su misión es múltiple, abarcadora de todos los campos en que puede mostrar su fecundidad el ingenio humano: "Un conjunto de hombres sabios, celosos por sus respectivas ciencias y mutuamente rivales, se ven llevados, por trato familiar y a favor de la paz intelectual, a armonizar las pretensiones y relaciones de sus disciplinas. Aprenden así a respetarse, tenerse en cuenta y ayudarse unos a otros. Se origina en consecuencia una atmósfera pura y clara de pensamiento, que también respiran los alumnos aunque éstos persigan sólo unas ciencias determinadas de entre toda una multitud. El estudiante se beneficia de una tradición intelectual, que es independiente de profesores individuales y que le guía en la elección de sus asignaturas e interpreta adecuadamente para él las que elige. Aprehende las grandes líneas del saber, los principios en los que descansa, las proporciones de sus diversas partes, sus luces y sombras, sus grandes y sus pequeños puntos, como de otro modo no lo aprehendería. Por eso se llama liberal a esta educación. Se forma con ella un hábito de la mente que dura toda la vida y cuyas características son libertad, sentido de la justicia, serenidad, moderación y sabiduría... Este es el fin principal de una universidad en el trato con sus estudiantes" (Cfr. Discursos sobre el fin y la naturaleza de la educación universitaria, $p$. 125. El destacado es nuestro).

De manera muy distinta al modo en que la Universidad alemana concibe sus fines, Oxford y Cambridge generan una tradición en la que no es la investigación lo que constituye la misión propia de los estudios sino que por encima de todo debe estar el aprendizaje. No es el progreso de los conocimientos, es la conservación y transmisión del saber lo propio de la actividad universitaria: "Advertimos que hay otras instituciones mucho más aptas como instrumento para estimular la investigación intelectual y extender las fronteras del saber que una universidad. Las universidades se establecen para enseñar las ciencias a los alumnos que allí se forman. Las academias se proponen realizar nuevas investigaciones para el progreso de las ciencias... Descubrir y enseñar son funciones diferentes. Obedecen también a dones distintos y generalmente no aparecen unidos en una misma persona. Quien dedica su día a transmitir el saber que posee, difícilmente tiene tiempo para adquirir saber nuevo... Los grandes descubrimientos en química y electricidad no se hicieron en universidades. Los observatorios astronómicos se hallan más frecuentemente fuera de las universidades que dentro de ellas..." (Cfr. Discursos..., pp. 29-30)

Lo que la Universidad enseña es mucho más que la mera preparación profesional. Ella es, ante todo, un ambiente educativo en el que se convive, se respira una atmósfera 
común y se busca la formación integral de la personalidad de los alumnos. No basta con impartir conocimientos, hay que crear hábitos de disciplina y solidaridad, afinar la capacidad de discernimiento, el sentido de la moderación, la conciencia del deber... La Universidad inglesa apunta a la formación del gentleman y por eso en su curricula incluye el deporte en tanto que ayuda a desarrollar ciertos rasgos indispensables de caballerosidad y de dignidad: "De ahí que casi sea una definición del gentleman el decir que lo es quien nunca da un pesar... El verdadero gentleman evita con todo esmero cuanto pueda provocar choque o sacudida en el espíritu de quienes están en contacto con él, todo roce de opinión o colisión de sentimiento, todo cohibimiento o sospecha, reserva o resentimiento, pues su gran cuidado es hacer que cada cual se sienta bien y a sus anchas... Quita toda importancia a los favores que hace y parece recibirlos cuando los otorga... Nunca habla de sí más que forzado, nunca se defiende con una mera retorsión, nunca de oídos a la calumnia o murmuraciones, es escrupuloso para imputar móviles a quienes se atraviesan a su paso, y a todo busca la interpretación mejor... Es paciente, sufrido y resignado... En ninguna parte encontraremos más sinceridad, consideración, indulgencia... Incluso si no es creyente, es demasiado profundo y abierto de espíritu para reirse de la religión o ir contra ella..." (Cfr. SALINAS, Universidad. y bien común, p.105. El destacado es nuestro).

La formación, que determina esencialmente la racionalidad del hombre en su totalidad, constituye una actividad típicamente humana. El buen sentido, la capacidad de juicio y la prudencia son aspectos fundamentales del saber liberal que debe ser el objetivo primario de la Universidad. La originalidad de Newman estriba en que, sobre estas bases generales y afines a cualquier intelecto cultivado, la Universidad que propugna tiene en cuenta la dimensión espiritual y sobrenatural de la persona. Es que Newman no habla sólo para el caballero culto sino que piensa en el hombre cristiano.

\section{Libertad academica. Universidad y Estado}

Como institución corporativa, la universidad pretendió el monopolio de la enseñanza y, a la vez, la independencia respecto de los poderes civiles. Los universitarios vivían en una ciudad "extraña", amparados por el derecho de los Pontífices y de los Reyes. Por eso se entiende que París y Bolonia nacieran en medio de los pueblos pero que Oxford y Cambridge hicieran realidad un "campus". No obstante, los privilegios de los universitarios, el prestigio de sus maestros y la creciente importancia de la cultura en la dirección de los pueblos incidieron para que la universidad tuviera su lugar en el gobierno del mundo. Por ej.: Ante una consulta del Rey Carlos I, Salamanca tuvo que pronunciarse respecto de los derechos de España en el gobierno de las poblaciones autóctonas de las Indias Occidentales. No obstante, a veces, esa influencia de los universitarios fue causa de conflictos con los poderes políticos.

Hacia fines del siglo XVIII hizo crisis la vida social y política en Francia; al "antiguo régimen" se le reprochaba: absolutismo monárquico y privilegios de los nobles; lujo en las cortes por despilfarro de los tesoros públicos; arbitrariedad en la aplicación de las leyes; aumento de la desigualdad entre las clases sociales. Los intelectuales impulsaban un cambio de régimen y ese clima favoreció la Revolución del 14 de julio de 1789. Entre los principios destacados de la Revolución se encuentra la Declaración de los derechos del hombre y del ciudadano (1789) que sostiene la igualdad de todos los hombres; la libertad de opinión y de religión; el derecho inviolable a la propiedad privada; la igualdad ante la ley y el derecho a desempeñar cargos públicos. 
En el "antiguo régimen" había crecido el analfabetismo; la enseñanza elemental se reducía a la lectura del catecismo; el uso de castigos corporales era frecuente; la situación de los maestros era precaria... Los ideólogos de la Revolución crearon las bases políticas para que en educación se desarrollara el concepto de "instrucción pública". Dice Compayré (1911): "La ciencia de la educación no les es deudora de nuevos métodos; no completaron la obra de Locke, Rousseau y de La Chalotais. Pero fueron los primeros en tratar de organizar, legislativamente, un vasto sistema de instrucción pública. Es justo colocarlos en primer lugar entre los hombres a quienes se podría llamar los políticos de la educación..." (Cfr. LARROYO, op. cit., p. 476). En coherencia con esas ideas, Talleyrand (1754-1838) logró que en la Asamblea Constituyente de 1791 se aprobara expresamente que "se creará y organizará una instrucción pública, común para todos los ciudadanos, gratuita en todas las partes de enseñanzas indispensables para todos los hombres"

Podemos afirmar que el nacimiento de la República no fue fácil ni inmediato, hasta comienzos del siglo XIX Francia tuvo que enfrentar luchas internas y guerras con países vecinos. Es más, en 1799 el General Napoleón Bonaparte derrocó a las autoridades y, después de reorganizar la administración y las finanzas, se hizo nombrar Emperador (1804); Francia volvió de la República a la monarquía. Durante su gobierno creó la Universidad Imperial (ley del 6 de mayo de 1806) con el objeto de que los estudios superiores tuvieran la unidad que gozaban en otros países.

A partir de 1806 todo el sistema educativo fue confiado a la universidad. La política imperial estaba dirigida a asegurar la concordancia absoluta de las opiniones culturales, morales y políticas puesto que era necesario someter las conciencias al poder. El designio supremo era el de la unidad y por eso había que reconstruir la universidad y la educación de acuerdo con una concepción militar y autoritaria. Una férrea centralización hacía de la Universidad de París el eje de ese complejo sistema cuyas autoridades eran designadas por el Ministerio del Interior.

La conformación dada por Napoleón a la universidad francesa se inspiraba en la idea de hacer de ésta una institución puesta directamente al servicio del Estado con el objetivo de preparar a los profesionales requeridos por la sociedad. La enseñanza superior asumió un carácter marcadamente profesionalizante y la producción de conocimiento tuvo una fuerte dependencia de las políticas impulsadas desde el poder. Ello implicó algunas consecuencias como la independencia de las facultades en desmedro del conjunto de la institución y la creación de escuelas politécnicas o de formación docente al margen de la universidad.

La herencia napoleónica en la organización universitaria no quedó limitada al territorio de Francia o a los que formaron parte del Imperio. Así, a fines del siglo XIX, Menéndez Pelayo advertía sobre las consecuencias del plan de estudios centralista que había sido implementado en España a partir de 1845: "Se centralizaron los fondos de las universidades, se las sometió a régimen uniforme y desde aquel día la universidad, como persona moral, como centro de vida propia, dejó de existir en España. Le sustituyó la oficina llamada instrucción pública, de la cual emanaron programas, libros de texto, nombramientos de rectores y catedráticos, y hasta circulares y órdenes menadísimas sobre lo más trivial del régimen interno de las aulas. A las antiguas escuelas en las que el gobierno para nada intervenía sucedieron otras en las que el gobierno intervenía en todo, hasta en los pormenores de indumentaria y en el buen servicio de los bedeles... Nada más contrario a la generosa y soberbia independencia de que disfrutan las grandes instituciones docentes del mundo moderno, las universidades inglesas y alemanas" (Cfr. Historia de los heterodoxos, T. II. Cit. por Galino (1982) en Textos pedagógicos hispanoamericanos, pp. 1132 y ss. El destacado es nuestro) $)^{13}$. 
El modelo napoleónico también se reflejó en las universidades hispanoamericanas en la época de los movimientos independentistas. Krotsch pone de ejemplo a la Universidad de Chile fundada por Andrés Bello (1842) sobre la antigua Universidad de San Felipe (1738) y dice que estas instituciones ya no se limitarán a formar en las profesiones que necesitaban las nacientes repúblicas sino que también se harán cargo del desarrollo científico y de la organización de los sistemas educativos.

Este es el modelo que, entre nosotros, encarnó la Universidad de Buenos Aires (1821). La misión cultural y científica y la libertad académica se subordinarán a la formación de profesionales. Su organización tendrá un desarrollo más ligado con las necesidades locales y, por eso, su enseñanza se apartará en parte de las tradiciones de la universidad colonial. Afirma Halperín Donghi (1962): "Desde antes de su fundación, la Universidad de Buenos Aires ve amenazado su destino como centro de saber por las urgencias inmediatas de la sociedad en la que nace, que exige de ella, antes que una actividad científica real, el cumplimiento de ciertas funciones sociales que el progreso de Buenos Aires hace ineludibles: el abandono de una tradición universitaria que se remonta a la Edad Media y se consolida en la España de la Contrarreforma no significa, entonces, para la Universidad de Buenos Aires, la adopción de una actitud más moderna frente a los problemas del conocimiento, sino un abandono del interés por ese problema" (Cfr. Historia de la UBA, p. 17).

\section{Modelo academico norteamericano ${ }^{14}$}

Las primeras instituciones norteanericanas reprodujeron la organización de las universidades inglesas de Oxford y Cambridge, muy comprometidas en la formación del clero protestante. Los primeros 'colleges' tenían orientación religiosa y se dirigían a la formación de una pequeña elite.

A fines del siglo XVIII la educación superior experimentó una notable expansión aunque los currículos de formación, centrados en los estudios clásicos y en las lenguas, no sufrieron cambios significativos. Nuevos 'colleges' se fundaron en ciudades nuevas para expresar el sentimiento de igualdad de la sociedad norteamericana y facilitar el acceso de las clases medias y rurales a los estudios de nivel superior. La mayor parte de esas instituciones surgieron gracias a la iniciativa de comunidades religiosas que tuvieron como referencia los curriculos de Harvard y Yale.

La aparición de universidades públicas y el establecimiento de relaciones entre la investigación y el desarrollo agrícola e industrial se produjo, sobre todo, desde la firma del Acta Morrill (1862) que confirió grandes extensiones de tierras fiscales a cada uno de los Estados para sustentar la educación superior. Según el concepto de 'land-grant' los límites de la Universidad se identificaban con los límites del Estado y, por ello, la institución debía ofrecer servicios a toda la población no sólo a través de la educación tradicional sino también por medio de la investigación aplicada al desarrollo de la industria y de la agricultura. De esa manera, el paradigma universitario cambió en un doble sentido: a) Los estudios científicos y clásico-liberales fueron desplazados por las enseñanzas de tipo práctico y b) Las instituciones comenzaron a preocuparse por ofrecer programas de estudios vinculados a las necesidades de la comunidad. De esa manera, el estudio y el trabajo fueron considerados como dos medios complementarios en la formación integral de las personas. En el saber hacer el hombre demuestra su vocación de servicio por los demás y justifica su actividad teorética puesto que el trabajo se humaniza cuando el hombre sabe por qué y para qué hace las cosas. 
Ejemplos de esta tradición fueron las Universidades de Wisconsin, Michigan, Illinois, Indiana, Minnesota y otras que crecieron rápidamente y se convirtieron en las primeras "multi-universidades" por la cantidad de servicios que combinaban: el servicio directo a la sociedad; la educación liberal como fundamento de la formación de grado y el énfasis puesto en la investigación como parte de la empresa académica.

A comienzos del siglo XX, gracias al viaje de muchos estudiantes norteamericanos, se reprodujo el modelo alemán de desarrollar la investigación como servicio para el crecimiento industrial. Hacia 1910 se consolidó la estructura básica de instituciones que priorizaron la investigación aunque la mayor expansión de las grandes multi-universidades se produjo entre 1950-1970. Desde entonces no se produjeron grandes cambios excepto la aparición de los "community colleges".

"Por todo esto, podemos decir que la universidad norteamericana contemporánea se forjó a partir de tres influencias: la tradición inglesa de las artes liberales, el concepto alemán sobre la investigación y la síntesis de estos elementos con la idea de servicio, encarnada en las universidades estatales". (ALBATCH, p.127). En esta red de instituciones existe una jerarquía y son las universidades de mayor prestigio, orientadas a la investigación, las que dominan sobre las demás y fijan las pautas curriculares. Las universidades privadas son libres para desarrollarse de manera independiente mientras puedan conseguir los recursos necesarios para su funcionamiento.

En la actualidad, el sistema universitario norteamericano aparece como bastante anárquico. Cuenta con más de trece millones de estudiantes post-secundarios y con más de tres mil instituciones: colleges y universidades. Las más importantes son unas cien universidades que se dedican a desarrollar la investigación y absorben gran parte de los recursos de origen privado y estatal. Sus profesores tienden a dominar las redes de conocimiento nacional e internacional. Sus tareas más importantes se realizan a nivel de posgrados aunque también ofrecen carreras de grado en diversas áreas científicas. Ej.: Harvard, Berkeley, Wisconsin... No obstante, advierte Francis Mollet Rogers: "La tradición europea, que los EE.UU. adoptaron, requiere que la Universidad sea un gran centro de investigación y el profesor una autoridad en su materia, autor de artículos, monografías, libros, en otras palabras, que sea un especialista. Pero ahora, dicha tradición tropieza con la necesidad de enseñar en forma efectiva. No todas las mil novecientas instituciones de educación superior pueden ser centros de investigación y sí buenos centros docentes o de enseñanza" (Cit. por NASSIF, op. cit., p. 317).

Una segunda categoría de instituciones está conformada por los colegios de artes y ciencias para la formación de grado. Algunas sólo se dedican a ofrecer programas de estudios de cuatro años a gran cantidad de estudiantes. Por lo general, ofrecen una educación básica en artes liberales con alguna clase de especialización en un campo académico o vocacional al final del ciclo. Las universidades más prestigiosas, que mantienen las tradiciones de la educación liberal inglesa, también suelen ofrecer algunas carreras de maestría.

Otro sector importante en esta red de instituciones lo integran los community colleges que facilitan el acceso abierto a la educación superior. Se calcula que alrededor de la tercera parte de los egresados secundarios se inscribe en este tipo de instituciones. Ofrecen carreras cortas de dos años y otorgan una diplomatura. El currículo es variado pero prioriza la formación profesional aplicada a diversos campos que permiten el rápido empleo. Uno de los principales propósitos de estas instituciones es ofrecer un medio de movilidad social y ocupacional a segmentos de la población de menores posibilidades. Los community colleges son, por lo general, instituciones públicas 
financiadas por los gobiernos locales y aunque sus egresados pueden continuar estudios en universidades de mayor nivel académico, esa transferencia de graduados es poco frecuente.

Si bien los EE.UU. no tienen una política educativa centralizada puesto que el gobierno y administración del sistema está a cargo de los Estados, en California se da una buena articulación en el nivel superior y todas las instituciones públicas definen su orientación, su modelo organizativo y su función de acuerdo con sus objetivos. La Universidad de California está en la cima de este sistema y ofrece diplomas doctorales y profesionales. Sus profesores, bien remunerados, dedican pocas horas a la enseñanza y priorizan la investigación y las publicaciones.

Durante el siglo XX el sistema académico norteamericano ha evolucionado hacia una combinación de estabilidad institucional conservadora, por un lado, y hacia la adaptación a nuevas tendencias, por otro. Esta evolución no ha sido fruto de un planeamiento sino de la misma evolución puesto que, ante un nuevo requerimiento, o bien se crean nuevas instituciones o se agregan funciones a las instituciones tradicionales sin cambiar esencialmente su organización. Las reformas institucionales a gran escala han sido poco frecuentes. Se ha respetado el considerable grado de autonomía de las universidades y se ha permitido que cada una de ellas produjera los cambios que consideraba necesarios en diversos grados y direcciones.

\section{Educacion Superior en Argentina}

Podemos comenzar este tema citando las palabras del Prof. Krotsch (2003, p. 187): "Si por origen entendemos la existencia de un acto inaugural que marca la historia posterior, como es el caso de la universidad humboltiana o napoleónica, podemos decir que la universidad argentina no tiene origen. La universidad argentina se desarrolla sobre la base aleatoria de distintos modelos prevalecientes en el mundo, al mismo tiempo que estos perfiles son moldeados y adaptados luego al estilo de demanda profesionalista que orienta a las clases medias en ascenso: proceso que se extiende a lo largo de todo este siglo y continúa teniendo vigencia aún hoy, y que posiblemente la exigencia de eficiencia y calidad de mercado no hará más que incrementar”.

Tanto Córdoba que reprodujo el modelo hispano-escolástico; como Buenos Aires inspirada en el modelo napoleónico; como La Plata que quiso adaptar el modelo de investigación científica tuvieron un sello común: su función principal fue la formación de profesionales. La expansión de la matrícula universitaria de la década del cincuenta y la creación de la Universidad Obrera ¿acaso no perseguían, también, el acceso a mejores puestos de trabajo y la movilidad social por medio de la formación profesional?. La fundación de universidades privadas en la década del sesenta ¿pudo ofrecer cambios a las estructuras tradicionales diseñadas por las universidades históricas?.

Sin embargo, como decía el Rector Fundador de la UNCuyo, Dr. Edmundo Correas (1940): "la universidad no puede limitarse a ser una fábrica de profesionales". La Universidad debe realizar la doble función de la formación profesional especializada y de la formación general. Eso es lo que manda la LES (1995): "Es finalidad de las instituciones universitarias la generación y comunicación de conocimientos del más alto nivel en un clima de libertad, justicia y solidaridad, ofreciendo una formación cultural interdisciplinaria dirigida a la integración del saber así como una capacitación científica y profesional específica para las distintas carreras que en ellas se cursen, para beneficio del hombre y de la sociedad a la que pertenecen" (art. 27).

Podemos preguntarnos: ¿a qué insistir con la necesidad de la dimensión teórica de los estudios universitarios?, ¿acaso el estudiante que ingresa a la Universidad no busca 
formarse en una determinada profesión?, ¿por qué no puede consistir la esencia de los estudios universitarios en la preparación de hábiles médicos, químicos o abogados?. En realidad, si esa formación quiere ser verdaderamente universitaria, si quiere realizarse en un clima de libertad, sin estar subordinada a ulteriores finalidades prácticas, debe ser académica. Naturalmente, la habilidad profesional del médico o del abogado debe ser un objetivo deseado pero esto necesita ese "plus" de aquel que supera la medianía y el aprendizaje de una técnica determinada. La diferencia, en el nivel universitario, está en dirigirse hacia las cosas superando las circunstancias particulares que las rodean para penetrar la insondable profundidad del mundo a la vista del carácter misterioso del ser. Es probable que sólo desde esta perspectiva podamos decir que el modelo de formación de profesionales no es una degradación del modelo original de Universidad.

Lo distintivo es estar libre de cualquier fin utilitario y en eso consiste la libertad académica; las ciencias particulares sólo pueden ser libres cuando son tratadas sin referencia a ningún fin utilitario. ¿Será ésta la actitud que nos reclama Gago Huguet (1995) en su conferencia "A pesar de todo"?: "El desafio fundamental de nuestras universidades sólo está de paso por los problemas del dinero insuficiente, del personal descalificado, de los rutinarios planes de estudio, de la investigación irrelevante, de las bibliotecas y centros de información caducos, del gasto ineficiente y muchos otros para llegar al problema central, el de las actitudes. El verdadero desafío es cambiar las actitudes y los comportamientos cotidianos de cada protagonista del quehacer educativo..." (Cfr. "La Universidad Latinoamericana ante los nuevos escenarios de la Región. Méjico: Universidad Iberoamericana-UDUAL. El destacado es nuestro).

Es probable que no todos los jóvenes tengan vocación o condiciones para emprender estudios universitarios. Para aquellos que sólo desean un título profesional de nivel superior, una carrera corta que habilite para desempeñarse en el mundo del trabajo, la LES 24521 ha previsto la creación de Institutos Superiores de Formación Docente y de Formación Técnica. Estos Institutos, de gestión estatal o privada, dependen de los gobiernos jurisdiccionales y, según el modelo norteamericano, pueden ofrecer carreras que satisfagan la demanda de egresados secundarios que por distintos motivos no pueden ingresar en la Universidad. Esas carreras pueden ser a término y deben tratar de atender a requerimientos concretos de las sociedades locales. Los estudios cursados en los Institutos Superiores deben tener un nivel de calidad equivalente y deben estar articulados con los estudios de grado y de posgrado que sólo pueden ofrecer las Universidades. ${ }^{15}$

En Políticas para el cambio y el desarrollo de la educación superior (1995), la UNESCO propone la realización de un pacto académico que mejore las condiciones de la Educación Superior en sus Estados miembros a fin de responder a las necesidades actuales y futuras del desarrollo humano sostenible. Considera que las políticas de educación superior latinoamericanas deben responder a cuatro tendencias: pertinencia; investigación; calidad e internacionalización puesto que en la actualidad se deben estudiar problemas complejos que no pueden ser abarcados por una sola disciplina científica (ej.: investigación genética; deuda internacional; derechos humanos; progreso tecnológico médico y militar; inteligencia artificial, etc.).

Se proponen las siguientes características para organizar una institución proactiva:

"El objetivo de la acción, en la que deben participar todos los interesados, es lograr que cada centro de educación superior se convierta en:

- Un lugar de formación de alta calidad que capacite a los alumnos para actuar de manera eficiente y eficaz en una amplia gama de funciones y actividades cívicas y profesionales, incluyendo las más diversas, actuales y especializadas. 
- Un lugar al que el acceso dependa principalmente del mérito intelectual y de la capacidad de participar activamente en sus programas, cuidándose de asegurar la igualdad social.

- Una comunidad dedicada plenamente a la investigación, la creación y la difusión del conocimiento, al progreso de la ciencia y que participe en el desarrollo de innovaciones e invenciones tecnológicas.

- Un lugar de aprendizaje basado únicamente en la calidad y el conocimiento, que inculque a los futuros graduados el compromiso de proseguir el aprendizaje y la responsabilidad de poner su formación al servicio del desarrollo social.

- Un lugar que acoja a los que regresan para actualizar y perfeccionar sus conocimientos y sus calificaciones como parte de la práctica y de la cultura institucional.

- Una comunidad en que se estimule y apoye activamente la cooperación con la industria y los servicios a favor del progreso económico de la Región y la Nación.

- Un lugar en el que se individualicen, discutan y aborden con espíritu de crítica bien informada problemas y soluciones locales, regionales, nacionales e internacionales importantes y en el que se fomente la participación activa de los ciudadanos en los debates sobre el progreso social, cultural e intelectual.

- Un lugar al que gobiernos y demás instituciones públicas puedan dirigirse en busca de información científica fiable, cada vez más necesaria para las autoridades de todos los niveles y que también promueva la participación en los procesos de decisión.

- Una comunidad cuyos miembros, íntegramente dedicados a los principios de libertad académica, estén comprometidos en la búsqueda de la verdad, la defensa y el fomento de los derechos humanos, la democracia, la justicia social y la tolerancia en sus propias comunidades y en todo el mundo, y que participan en la instrucción encaminada a la verdadera ciudadanía participativa y en la edificación de una cultura de paz.

- Una institución bien ubicada en el contexto mundial con todas las amenazas y las posibilidades inherentes y adaptada al ritmo de la vida contemporánea, a las características distintivas de cada región y de cada país”. (El destacado es nuestro)

$¿$ No reconocemos en algunas de estas propuestas un retorno al ideal primitivo de la Universidad entendida como comunidad de maestros y estudiantes que buscan la sabiduría?. Sin ignorar la importancia que reviste la vinculación con los problemas sociales, nos animamos a afirmar que el futuro de nuestras instituciones dependerá principalmente de la seriedad y responsabilidad con que los universitarios desempeñemos nuestra vocación intelectual.

\section{Bibliografía y fuentes}

ALTBACH, Philip (2001). Educación superior comparada. El conocimiento, la universidad y el desarrollo. Trad. de Adelaida Ruiz. Bs. As.: Universidad de Palermo.

CATURELLI, Alberto (1963). La Universidad. Su esencia, su vida, su ambiente. Córdoba: Dirección general de publicaciones de la Universidad Nacional.

CIRIGLIANO y AMEGHINO (1970). El poder joven. Bs.As.: Libr. de las Naciones.

CORVALAN LIMA, Héctor (1983). La universidad en el acontecer nacional. Mendoza: Editorial Idearium de la Universidad de Mendoza.

DERISI, Octavio (1980). Naturaleza y vida de la universidad. $3^{\circ}$ ed. Buenos Aires: Editorial El Derecho. Universidad Católica Argentina. 
GALINO, Ma. Angeles (1973). Historia de la Educación: Edades Antigua y Media. $2^{\circ}$ ed. Madrid: Edit. Gredos.

INFORME DELORS (1996). La educación encierra un tesoro. Madrid: Santillana.

KROTSCH, Pedro (2003). Educación superior y reformas comparadas. Colección Cuadernos Universitarios, $\mathrm{N}^{\circ}$ 6, Universidad Nacional de Quilmes.

LARROYO, Francisco (1981). Historia general de la Pedagogía. $17^{\circ}$ ed. México: Editorial Porrúa.

MONTEJANO, Bernardino (1979). La Universidad. Buenos Aires: Edic. Ghersi.

NASSIF, Ricardo (1984). Teoría de la educación. Problemática pedagógica contemporánea. $2^{\circ}$ reimpresión. Madrid: Cincel-Kapelusz

NEWMAN, John (1996). Discursos sobre el fin y la naturaleza de la educación universitaria. Trad. de José Morales. Navarra: EUNSA.

PIEPER, Josef (1979). El ocio y la vida intelectual. Trads. de Masegosa; Salcedo; García Ortega y Cercós. Madrid: Rialp S.A.

PIEPER, Josef (1989). En defensa de la Filosofía. $6^{\circ}$ ed. Trad. de Alejandro Laton Ros. Barcelona: Edit. Herder.

RANDLE, Patricio (1974). La universidad en ruinas. Bs. As.: Edit. Almena.

RODRIGUEZ CRUZ, Agueda (1992). La universidad en la América Hispánica. Madrid: MAPFRE S.A.

SILES SALINAS, Jorge (1972). La universidad y el bien común. La Paz, Bolivia: Universidad Mayor de San Andrés.

TAQUINI (h), Alberto (2000). La transformación de la educación superior argentina: de las nuevas universidades a los colegios universitarios. Buenos Aires: Academia Nacional de Educación.

UNESCO (1995). Políticas para el cambio y el desarrollo en la educación superior. París.

VOCOS, Francisco (1981). El problema universitario. $2^{\circ}$ ed. Buenos Aires: Colección Ensayos Doctrinarios.

\footnotetext{
${ }^{1}$ Cfr. Montejano (h) (1979), La Universidad, p. 96. En la misma línea, podemos mencionar a Josef Pieper; Karl Jaspers; Francisco Vocos; Nicolás Derisi; Alberto Caturelli y muchos otros profesores universitarios.

${ }^{2}$ Para comparar el conocimiento académico del filósofo con el del científico positivista, Pieper (1989) cita un párrafo de Carnap (1931): "Todo se reduce a superficie; todo es accesible al hombre. Es una aberración preguntar siquiera por una raíz de las cosas, y sobre todo por su última razón y significado. En una palabra: no existe en absoluto ese misterioso objeto de la filosofía. Sólo hay objetos de la ciencia, y en todo rigor éstos son, sin excepción, objeto de la física" (Cfr. En defensa de la Filosofía, p 20 y ss.)

${ }^{3}$ El Prof. Krotsch (2003) cita a Durkheim que afirma la importancia de estudiar los orígenes de las instituciones, de reconstruir el pasado, pues "para entender bien el desarrollo de un ser vivo, para explicar las formas que presenta en los momentos sucesivos de su historia, habría que comenzar por conocer la constitución del germen inicial que es el punto de partida de toda su evolución..." (Cfr. Educación superior y reformas comparadas, p. 57)

${ }^{4}$ El estudio de la Filosofía se denomina "artes liberales" porque supone la necesaria libertad que debe tener el espíritu para investigar y buscar la verdad donde se encuentre. El hombre libre no está esclavizado en las sombras de lo cotidiano y no busca la "utilidad" como objetivo de su trabajo intelectual; esto es propio de las "artes mecánicas"

${ }^{5}$ Severino Boecio (480) fue un noble romano que estudió en Roma, Atenas y Alejandría. A este filósofo se atribuye la clásica definición de persona como individua substantia, rationalis naturae. Fue un personaje que tuvo influencia en la política de su tiempo y que fue condenado a muerte. Durante sus últimos días en prisión escribió su obra: Consolación de la Filosofía.

${ }^{6}$ Aunque en Bolonia ya se estudiaba Derecho y en Salerno Medicina; para Montejano (1979) sólo París tiene que ser reconocida como primera universidad porque allí se estudiaba Filosofía y Teología. Se apoya en un argumento de Carlos Sanz que dice: "la Universidad sólo es tal, si se da esa apertura científica hacia el
} 
primer principio y causa última de las cosas. No puede, por ende, quedarse en una perspectiva meramente técnica, ni en lo estrictamente científico, sino que, por el contrario, debe integrar los saberes particulares a la luz de la sabiduría. Actitud sapiencial que, llevada hasta las últimas consecuencias, no puede terminarse tampoco en un planteamiento metafísico o de teodicea, sino que debe llegar hasta los principios revelados" (Cfr. La Universidad, p. 77).

${ }^{7}$ Entre esos maestros, Krotsch otorga una importancia especial a Abelardo porque era capaz de organizar grandes debates en torno a la escolástica “... fue él quien atrajo a multitudes de estudiantes en torno a un espacio educativo concreto como fue Notre Dame..., lugar en torno al cual se constituirá un centro de atracción y referencia, de concentración de la vida espiritual y cultural que será el núcleo del que emergerá la universidad" (Cfr. op.cit., p.61).

${ }^{8}$ Según Wilson (1965): "Durante todo el siglo XIII encontramos en la Facultad de Artes de París un conjunto de profesores que sólo piden libertad para enseñar la lógica, la física y la moral de Aristóteles sin preocuparse de otras disciplinas ni de los intereses superiores de la teología. El averroísmo parisiense es la forma más visible y la manifestación más brutal de esta tendencia. Ante ella, aparece la Facultad de Teología, rigurosamente agustiniana. El término medio superador lo encontramos en la intuición genial de Alberto Magno y de Santo Tomás de Aquino, y el secreto de su triunfo absoluto consistirá precisamente en que armonizarán las dos tendencias divergentes, e incluso contradictorias, en que se dividía la Universidad de París, legitimando todo el contenido positivo con que venía a enriquecerse la enseñanza de las Artes liberales y organizando, desde este punto de vista, el edificio de la teología tradicional, más acabado y sólido en adelante que hasta entonces" (Cfr. La Filosofía en la Edad Media. Cit. por MONTEJANO, op.cit., p.88)

${ }^{9}$ Pérez del Viso (1968) decía respecto de la estructura de la Universidad de Bolonia: “El profesor es, por de pronto, una especie de docente contratado por un grupo de caballeros independientes entre los 17 y los 40 años. Estos estudiantes no deben a sus profesores obediencia espiritual de ninguna clase; como dueños de su universidad, los estudiantes ejercen considerable presión sobre la ciudad y sobre los profesores, negocian con la ciudad sobre los precios de la vivienda y los comestibles e intervienen en los constantes litigios jurídicos" (Cit. por CIRIGLIANO (1970) en El Poder Joven, p. 34).

${ }^{10}$ Cfr. art. "Oxford" en Rev. Vida Universitaria, $\mathrm{N}^{\circ}$ 10, septiembre de 1965, Bs. As. (Cit. por Montejano, op.cit., p. 85 y ss.)

11 "La Universidad es una escuela, pero escuela única en su género. En ella no sólo se debe enseñar, el alumno debe participar en la investigación y llegar así a una formación científica decisiva para su vida. De acuerdo con la idea, los alumnos son pensadores independientes, autorresponsables, que siguen con espíritu crítico a su maestro. Poseen la libertad de aprender" (Cfr. Jaspers, La idea de universidad. Cit. por CIRIGLIANO, op.cit., p. 39).

12 El Prof. Krotsch (2003) compara la diferencia de nacimiento entre las universidades europeas y las hispanoamericanas. Aquellas surgieron como productos del desarrollo social y económico; de la creciente movilidad de estudiantes y maestros o como resultado de nuevas ideas. En cambio, en Hispanoamérica serán parte de un proceso de trasplante cultural. "Serán en lo fundamental, producto de un hecho administrativo que al mismo tiempo respondía a distintas orientaciones espirituales, como el humanismo que caracterizó a la Universidad de México o la conquista espiritual a través de la universidad misionera de los jesuitas, que no dejaba de expresar bajo el mandato de la contrarreforma el espíritu disciplinario que exigía el naciente orden burgués" (Cfr. op. cit., p. 124)

13 Afirma el que fuera Rector de la Universidad de Mendoza, Héctor Corvalán Lima (1983): "En los comienzos de la Edad Moderna, con la prevalencia absoluta del principio político y la consiguiente aparición del absolutismo monárquico, es cuando el Estado va a intentar, en forma sistemática, instrumentar a la Universidad para sus fines políticos. Esta ingerencia va a asumir una peligrosa dimensión después de la Revolución Francesa, cuando los Estados pretenden someter al credo surgido de la misma a todas las inteligencias, sobre todo en el recinto universitario, fuente de los futuros dirigentes políticos del país... Así el laicismo decimonónico va a estatizar toda la enseñanza en forma monopólica. Con un sentido claramente totalitario, van a ser excluídas de las tareas académicas todas aquellas instituciones que no sean directamente controlables por el Estado liberal. En los casos en que se permita la acción privada en el campo de la educación, ello va a ser incorporándola a la oficial e imponiéndole los programas y textos de estudio" (La universidad en el acontecer nacional, p. 76)

${ }^{14}$ Cfr. Altbach, Philip (2001); El sistema académico norteamericano desde una perspectiva histórica, pp.125 y ss.

15 Actualmente existen en Argentina alrededor de dos mil Institutos Superiores que ofrecen carreras profesionales de diversa calidad. En la medida en que puedan mejorar sus prácticas y ser más competitivos 
pueden ser una muy buena propuesta para responder a la demanda de muchos jóvenes que terminan la escuela secundaria. De esa manera se podría evitar la apertura de nuevas universidades nacionales que no están suficientemente preparadas para cumplir con eficiencia las funciones de investigación y docencia en el nivel de grado y de posgrado. Desde otra perspectiva, no sería irrelevante pensar que estas instituciones también podrían significar un importante ahorro de recursos.

Recebido em: $\quad 01 / 11 / 11$

Aprovado em: $\quad 04 / 02 / 12$ 